\title{
Near-field spectroscopy of cylindrical phonon-polariton antennas
}

\author{
Andrea Mancini, ${ }^{*, \dagger}$ Christopher R. Gubbin, ${ }^{\ddagger}$ Rodrigo Berté, ${ }^{\dagger}$ Francesco \\ Martini, ${ }^{\ddagger}$ Alberto Politi, ${ }^{\ddagger}$ Emiliano Cortés, ${ }^{\dagger} \mathrm{Yi} \mathrm{Li}^{\S}{ }^{\S}$ Simone De Liberato ${ }^{\ddagger}{ }^{\ddagger}$ and \\ Stefan A. Maier ${ }^{\dagger}$ \\ $\dagger$ Chair in Hybrid Nanosystems, Nanoinstitute Munich, Faculty of Physics, \\ Ludwig-Maxilimians-Universität München, 80539 München, Germany \\ $\ddagger$ School of Physics and Astronomy, University of Southampton, Southampton, SO17 1BJ, \\ United Kingdom \\ 【Istituto di Fotonica e Nanotecnologie - CNR, Via Cineto Romano 42, 00156 Roma, Italy \\ $\S S$ chool of Microelectronics, MOE Engineering Research Center of Integrated Circuits for \\ Next Generation Communications, Southern University of Science and Technology, \\ Shenzhen 518055, China
}

E-mail: A.Mancini@physik.uni-muenchen.de

\begin{abstract}
Surface phonon polaritons (SPhPs) are hybrid light-matter states in which light strongly couples to lattice vibrations. SPhPs exist inside the Reststrahlen band of polar dielectrics, where the dielectric function of the material becomes negative due to the presence of optical phonons in the infrared region. SPhPs antennas easily outperform their plasmonic counterparts in terms of field enhancement and confinement thanks to the inherently slower phonon-phonon scattering processes governing SPhPs decay.
\end{abstract}


In particular, SPhPs antennas have attracted considerable interest for thermal management at the nanoscale, where the emission strongly diverts from the usual far-field blackbody radiation due to the presence of evanescent waves at the surface. However, far-field measurements cannot shed light on the behavior of antennas in the near-field region. To overcome this limitation, we employ scattering-scanning near field optical microscopy (sSNOM) to unveil the spectral near-field response of 3C-SiC antenna arrays. We present a detailed description of the behavior of the antenna resonances by comparing far-field and near-field spectra. Furthermore, we investigate the perturbation in the antennas response induced by the presence of the AFM tip with the help of 3D electromagnetic simulations.

\section{Introduction}

Concentrating electromagnetic (e.m.) fields on deeply subwavelength scales via metal nanostructures has driven the field of plasmonics in recent decades. ${ }^{1-3}$ E.m. field confinement is achieved by the coupling of light with collective excitations of conduction electrons, resulting in strongly coupled states named surface plasmon polaritons (SPPs), which exist in the spectral region where the real dielectric function $\epsilon_{1}(\omega)$ is negative. The most common plasmonic materials are noble metals, for which the resonance frequency for a small particle $(d \ll \lambda)$ embedded in a dielectric environment with permittivity $\epsilon_{\text {env }}$ lies in the visible range as determined by the Fröhlich condition $\epsilon(\omega)=-2 \epsilon_{e n v} \cdot{ }^{4,5}$ Noble metals are characterized by high losses at optical frequencies due to electron-electron scattering, which limits the typical lifetime of SPPs to tens of femtoseconds. ${ }^{6-9}$ Consequentially, many applications in which low losses is a necessity, such as waveguides, ${ }^{10-13}$ metamaterials, ${ }^{14-16}$ integrated plasmonic circuits ${ }^{17,18}$ or quantum information processing systems, ${ }^{19,20}$ have yet to be realized efficiently. ${ }^{21}$ At the same time, lossy plasmonic antennas have been successfully employed for applications in which absorption and its consequent hot-electron generation and temperature increase are of interest, as in thermoplasmonics, ${ }^{22,23}$ cancer treatment ${ }^{24}$ and photocatalysis. ${ }^{25-27}$ 
The need for low optical losses and strong field confinement has spurred interest in doped semiconductors, ${ }^{28-30}$ graphene ${ }^{31,32}$ and conducting oxides ${ }^{33,34}$ as alternatives to noble metal plasmonics. ${ }^{21,35}$ These material systems still rely on electrons to mediate photonic field confinement but have lower electron densities and typical operating frequencies lie in the near or mid-infrared (MIR). These systems have weaker electron-electron scattering than noble metals, but both operating frequency and damping rate scale proportionally and the modal quality factor $(Q \approx \omega / \gamma)$ is unimproved. ${ }^{21,36}$ At the same time, the infrared (IR) range is particularly interesting for the interaction with molecular vibrations, allowing the implementation of chemically selective nanophotonic sensors. ${ }^{37,38}$ Noble metals themselves can be successfully employed for IR applications, ${ }^{37,39}$ however in this limit the photon field is far from resonance with the electron gas and the field confinement is inefficient.

Polar dielectrics are an alternative material system for MIR light confinement. In these system, the optic phonon dipole moment leads to a region where the real dielectric function $\epsilon_{1}(\omega)$ is negative, termed the Reststrahlen band, which lies between the transverse optic (TO) and longitudinal optic (LO) phonon frequencies. ${ }^{36,40}$ Inside the Reststrahlen band, as for conventional plasmonic materials, localised and propagating resonances called surface phonon polaritons (SPhPs) exist. As SPhPs are collective excitations of polar lattice vibrations they are independent of electrons and their lifetime is limited by phonon-phonon scattering, taking place on the order of picoseconds. These longer lifetimes result in larger quality factors and smaller mode volumes than comparable plasmonic structures. These properties make SPhPs-based nanostructures excellent candidates for ultra-sensitive molecular detection ${ }^{41-43}$ or as antennas to enhance MIR emission. SPhPs have also been used to control thermal emission at the nanoscale, demonstrating very different behavior from blackbodies. ${ }^{44-48}$ These applications require knowledge of the near-field spectrum of the system under study, which cannot be easily inferred from far-field measurements. The near-field response of an optical system can be found by investigating it with scattering scanning near field optical microscopy (sSNOM).$^{4-51}$ 
(a)

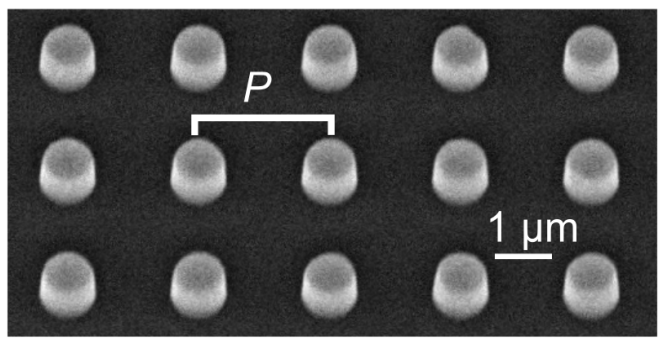

(b)

Experiment $\quad$ (c) Simulation
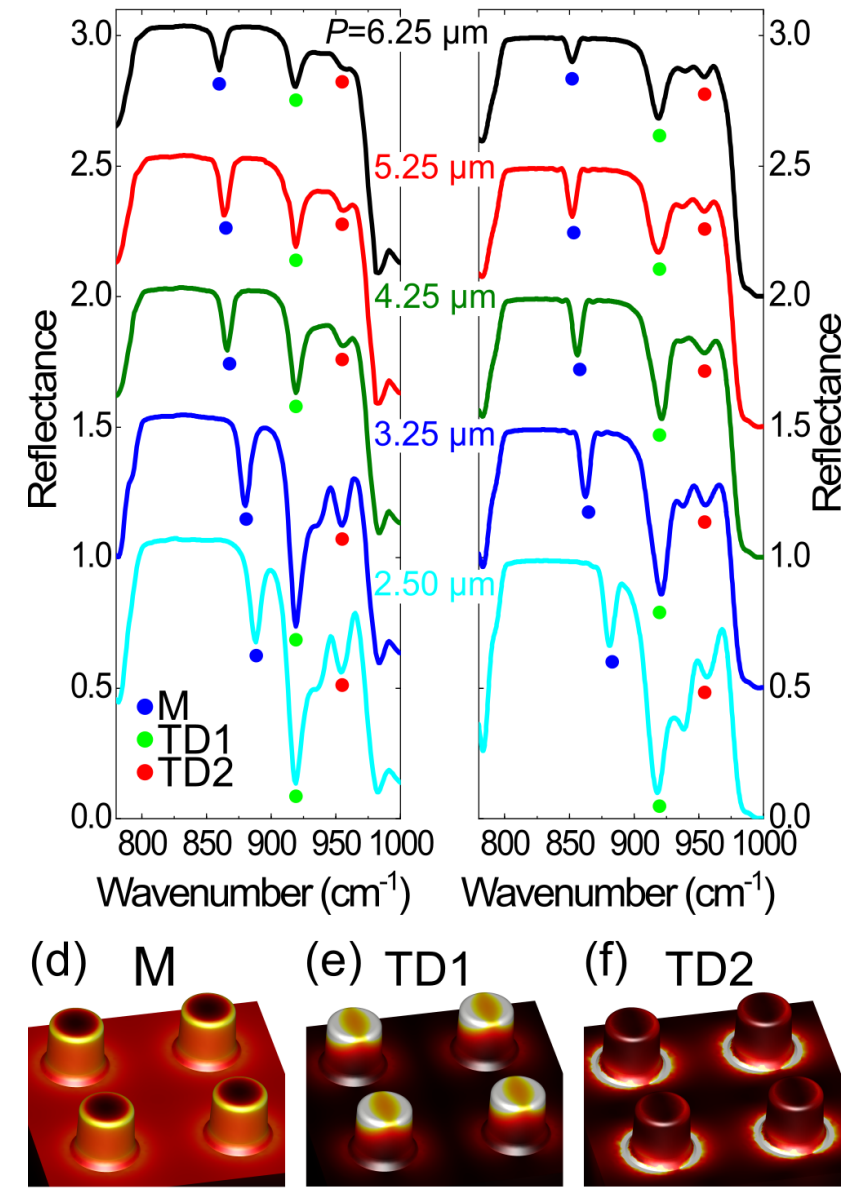

(e) TD1

(f) TD2
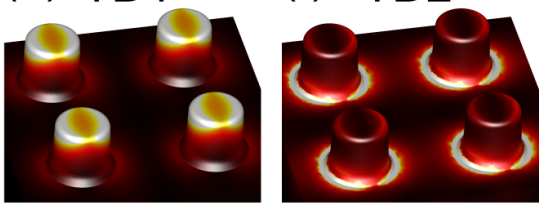

$\begin{array}{lllllll}0 & 10 & 20 & 30 & 1 & 40 & 50\end{array}$

Figure 1: (a) SEM image of a portion of the $\mathrm{SiC}$ pillar $(h \approx d \approx 1 \mu \mathrm{m})$ array with spacing $P=2.5 \mu \mathrm{m}$. (b) Experimental FTIR and (c) simulated spectra of the investigated arrays with different $P$. On the lower energy side, marked by blue dots, the monopolar mode (M) redshifts as $P$ increases due to reduced interpillar mode repulsion. At approximately $\omega_{T D 1}=920 \mathrm{~cm}^{-1}$ and $\omega_{T D 2}=955 \mathrm{~cm}^{-1}$ the first (TD1) and second (TD2) transverse dipolar modes are marked by green and red dots, respectively. Spectra are vertically shifted for clarity. Simulations of the field intensity at the surface for $P=2.5 \mu \mathrm{m}$ at the (a) monopolar $\omega_{M}=897 \mathrm{~cm}^{-1}$, (b) first transverse dipolar $\omega_{T D 1}=920 \mathrm{~cm}^{-1}$ and (c) second transverse dipolar $\omega_{T D 2}=954 \mathrm{~cm}^{-1}$ frequencies. Note how the field enhancement is much higher on the $\mathrm{SiC}$ substrate for the monopolar mode than for the transverse modes, leading to interpillar coupling even at $\approx 3 \mu \mathrm{m}$ distance. 
Here, we directly investigate the near-field spectral response of 3C-Silicon Carbide (SiC) pillars arrays by sSNOM. We show that the spectral response of the system in the near-field and far-field differ substantially and investigate to which degree this mismatch is related to the perturbation induced by the presence of the sSNOM AFM tip. We directly analyze the influence of the tip by employing 3D simulations where a metallic sphere modeling the tip is placed above an isolated $\mathrm{SiC}$ pillar. By scanning the tip over the surface we observe intensity variations of the array resonances both on the pillars and on the substrate between them. The understanding and control of the spectral near-field response we show in this paper opens the way to employment of $\mathrm{SiC}$ pillar arrays in MIR applications.

\section{Results and Discussion}

Arrays of SiC pillars ${ }^{52,53}$ have attracted considerable attention thanks to their ease of fabrication and rich e.m. response, with broadly tunable modes ${ }^{54}$ strong interaction between propagative and localized resonances, ${ }^{55}$ and nonlinear response. ${ }^{56-58}$ One limitation of SPhPs is the narrow frequency band in which they can be excited and efforts have been made to extend it with carrier injection. ${ }^{59} \mathrm{SiC}$ is particularly interesting thanks to its wide Reststrahlen band, extending from $\omega_{T O} \approx 800 \mathrm{~cm}^{-1}$ to $\omega_{L O} \approx 970 \mathrm{~cm}^{-1}$. The exact values of $\omega_{T O}$ and $\omega_{L O}$ vary depending on polytype. Additionally, the low optical losses of $\mathrm{SiC}$ make it one of the best materials for fabricating antennas supporting localized $\mathrm{SPhPs}^{36}$

As reported in more detail previously, ${ }^{54,55} \mathrm{SiC}$ pillar structures are fabricated by dry etching a 3C-SiC layer through a hard mask fabricated by standard e-beam lithography, so that the pillars are supported by a $\mathrm{SiC}$ substrate. The resulting pillar height and diameter are both around $1 \mu \mathrm{m}$, while the interpillar spacing $P$, defined as the distance between adjacent pillar centers, is varied from $2.5 \mu \mathrm{m}$ to $6.25 \mu \mathrm{m}$. In Figure 1 (a) a SEM image of a portion of one of the arrays $(P=2.5 \mu \mathrm{m})$ is shown.

The far-field optical response of the system is well understood ${ }^{53}$ by considering the res- 
onances of a single pillar coupled to the SPhPs of the bare SiC surface. The coupling can be described by the hybridization model, analogously to what happens for the resonance of plasmonic particles close to a metallic surface. ${ }^{60}$ The presence of the array structure also folds the dispersion of the bare $\mathrm{SiC}$ SPhPs, which can then lead to hybridization of localized and propagating modes. ${ }^{55}$ Of particular interest is the monopolar mode, arising from the coupling of the dipolar mode along the pillar height with the bare $\mathrm{SiC}$ substrate. The $\mathrm{SiC}$ substrate allows the charge of the monopolar mode to spread around the pillar base, generating coupling between adjacent pillars even at $\approx 3 \mu \mathrm{m}$ distance. ${ }^{54}$ The modes resulting from coupling of resonances polarized along the width of the pillar to the $\mathrm{SiC}$ surface have been named transverse dipole modes. ${ }^{52,54}$

We investigate the far-field optical response of the arrays with FTIR spectroscopy using a Bruker Infinion microscope in reflection mode with a 15-magnification Cassegrain objective, which illuminates the sample with a weighted average incident angle of $25^{\circ}$. All spectra are normalized with a reference spectrum obtained on a clean gold surface. In Figures 1 (b), (c) experimental and simulated far-field spectra of arrays with different $P$ are reported. Simulated spectra were obtained with a commercial solver (CST Studio Suite) and result from averaging TM and TE polarizations, as in experiments the light source is unpolarized, and have been smoothed in order to account for resonance broadening related to fabrication inhomogeneities. All spectra feature an increase in reflectivity corresponding to the $\mathrm{SiC}$ Reststrahlen band, while the resonances of the system appear as dips in this high-reflectivity wavelength range. The lower energy resonance, marked by blue dots in Figure 1 (b) and (c), can be identified as the monopolar mode, which redshifts with increasing $P$ due to reduced pillar repulsion. ${ }^{54}$ By green and red dots at $\omega_{T D 1}=920 \mathrm{~cm}^{-1}$ and $\omega_{T D 2}=955 \mathrm{~cm}^{-1}$ the first (TD1) and second (TD2) transverse dipole modes are marked respectively. In Figures 1 (d-f) field enhancements at the surface for the three main resonances are reported. The field distribution for the transverse dipole modes is much more confined at the pillar edges than for the monopolar mode. For this reason we do not see any significant shift for TD1 
and TD2 with varying $P$. The transverse dipole modes can also shift due to interpillar coupling, ${ }^{54}$ but only for values of $P$ much smaller then the one analyzed in this study. The small shoulder around $\omega=935 \mathrm{~cm}^{-1}$ can be attributed to a higher order transverse resonance, almost spectrally overlapped with the TD1 resonance.

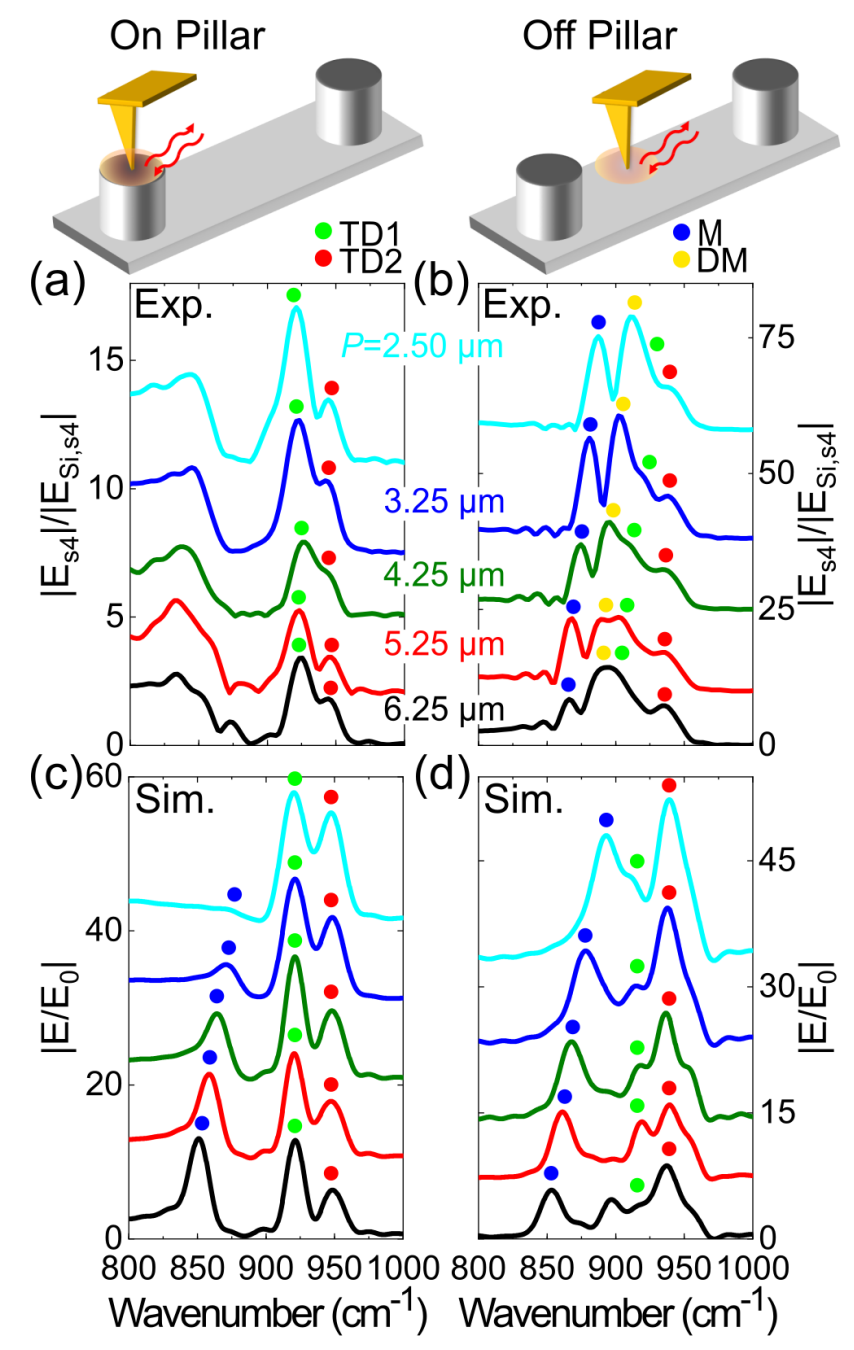

Figure 2: Near-field measurements of the spectral response for different array periodicity $P$. The spectra are collected either on (a) the center of a pillar, or (b) on the substrate inside the array at $\approx 800 \mathrm{~nm}$ from a pillar center as illustrated. The different modes are indicated with dots, using the same color code as in Figure 1: green for transverse dipole 1, red for transverse dipole 2, blue for the monopole and yellow for the dark monopole. Simulated near-field enhancement spectra at $5 \mathrm{~nm}$ above the surface on top of a pillar (c) and on the substrate between pillars (d), matching the measurement geometry. Spectra are vertically shifted for clarity.

The near-field response of the system was studied with a commercial sSNOM (Neaspec 
GmbH, Germany) equipped with a Michelson interferometer. The presence of the interferometer enables the measurement of IR spectra as in conventional FTIR. In the sSNOM (or nano-FTIR) arrangement, one of the two arms of the interferometer is comprised of a parabolic mirror which focuses and collects light backscattered from the AFM tip, ${ }^{50}$ while in the other a periodically moving mirror is used to produce interferograms from which spectra are obtained by Fourier transform. The resulting spatial resolution is much smaller than the diffraction limit, down to roughly the size of the tip radius of curvature (usually in the range of $20 \mathrm{~nm}$ to $40 \mathrm{~nm}$ ). To suppress far-field background contributions, the tip is vertically modulated at a tapping frequency $\Omega \approx 250 \mathrm{kHz}$ and the optical signal demodulated at higher harmonics $n \Omega$, being $n$ an integer number. The light source is a broadband pulsed laser (Toptica, Germany), emitting in the range $600 \mathrm{~cm}^{-1}$ to $1300 \mathrm{~cm}^{-1}$, covering the full extent of the $\mathrm{SiC}$ Reststrahlen band. The beam is incident at $\theta \approx 45^{\circ}$ with respect to the sample surface normal direction and is TM polarized to enhanced near-field scattering. ${ }^{61}$ In the following all measurements are demodulated at the fourth harmonic $4 \Omega$ and normalized to a reference spectrum obtained on a flat silicon surface $E_{\mathrm{s} 4, \mathrm{Si}} \mid$. We will indicate this in the near-field measurements label as $\left|E_{\mathrm{s} 4} / E_{\mathrm{s} 4, \mathrm{Si}}\right|$. Since it is well known that the presence of the AFM tip can perturb the optical response of strongly resonating antennas, ${ }^{62}$ we do not use standard nano-FTIR tips, instead choosing more conventional metal-coated AFM tips with smaller radius of curvature (Arrow-NCPt, $r<25 \mathrm{~nm}$ ). Uncoated (dielectric) tips were also considered, but the signal was too low for efficient normalization by a reference spectrum, leading to unreliable results (see Supporting Information).

To understand the near-field response of the system we probed with the AFM tip positioned either on the center of one of the pillars (position A) or on the substrate between the pillars at approximately $800 \mathrm{~nm}$ from the pillar center (position B), as illustrated in the sketch at the top of Figure 2. Results are reported in Figure 2 (a) and (b) for positions A and B, respectively. Spectra in Figure 2 (a) show that the response on the pillars is independent of the array spacing, since we can identify two peaks, marked with green and red 
dots, at $920 \mathrm{~cm}^{-1}$ and $945 \mathrm{~cm}^{-1}$ for all the arrays. These match well with the frequencies of the first (red) and second (green) transverse dipole modes measured in the far-field at $\omega_{T D 1}=920 \mathrm{~cm}^{-1}$ and $\omega_{T D 2}=955 \mathrm{~cm}^{-1}$. The monopolar mode is absent when measuring at position A, while it is detected when measuring on the substrate and it appears as a lower energy peak which redshifts with increasing $P$, as shown in Figure $2(\mathrm{~b})$. The frequency of the monopolar mode in the near-field is also found to closely match that reported in far-field measurements. Surprisingly at higher energies we see an additional mode, subject to similar redshifts, which cannot be associated with any far-field resonance. This is illustrated by yellow dots in Figure 2 (b). We associate this with a dark version of the monopolar mode which, as it has no net dipole moment, can be excited only in the presence of the near-field illumination provided by the tip. The broad peak below $850 \mathrm{~cm}^{-1}$ in Figure 2 (a) can be identified as a non suppressed far-field contribution, as can be inferred from from investigation of its amplitude as a function of increasing harmonic demodulation order $\mathrm{n} \Omega$ (see Supporting Information). Interestingly, the signal from transverse dipoles is also detected when measuring on position B, as shown by the presence of peaks above the monopolar mode in Figure 2 (b). Even though the frequency of the transverse modes is similar in Figure 2 (a) and Figure 2 (b), in the latter there is a small redshift with increasing $P$ especially for TD1 (green dot), indicating some weak interpillar coupling for the transverse modes when measured on the substrate. This feature is absent in the spectra collected at position A, indicating that this effect is mediated by the interaction with the tip.

In Figure 2 (c), (d) we report simulated spectra of the near-field amplitude at positions A and $\mathrm{B}, 5 \mathrm{~nm}$ above the pillar center and the $\mathrm{SiC}$ substrate respectively. In these simulations the incident angle of the light is set to $45^{\circ}$ to better represent the experimental conditions, but no modeling of the AFM tip is attempted. To take into account the finite spectral resolution of the interferometer, related to the maximum distance of $800 \mu \mathrm{m}$ traveled by the moving mirror (see Supporting Information), simulated spectra shown in Figures 2 (c), (d) have been smoothed with a FFT filter. Spectra simulated in this way only partially reproduce 
the measured spectra due to interaction between the AFM tip and the SPhP antennas. Nevertheless, we can see that for both simulations on and off pillars in Figures 2 (c) and (d), the TD1 and TD2 transverse modes, marked by green and red dots, lie close in frequency to the corresponding experimental data. Their relative amplitudes differ from the experimental values, and this could be attributed to the different illumination geometry and collection efficiencies, as the AFM tip predominantly backscatters the out-of-plane component of the field $E_{z}$. Strikingly, simulations do not show at all the dark monopole, marked with yellow dots in Figure 2 (b). This further suggests that this resonance is active only in the presence of the AFM tip, which acts as a near-field source, allowing the excitation of modes with no net dipole moment. We investigate this further by 3D simulations, as follows.
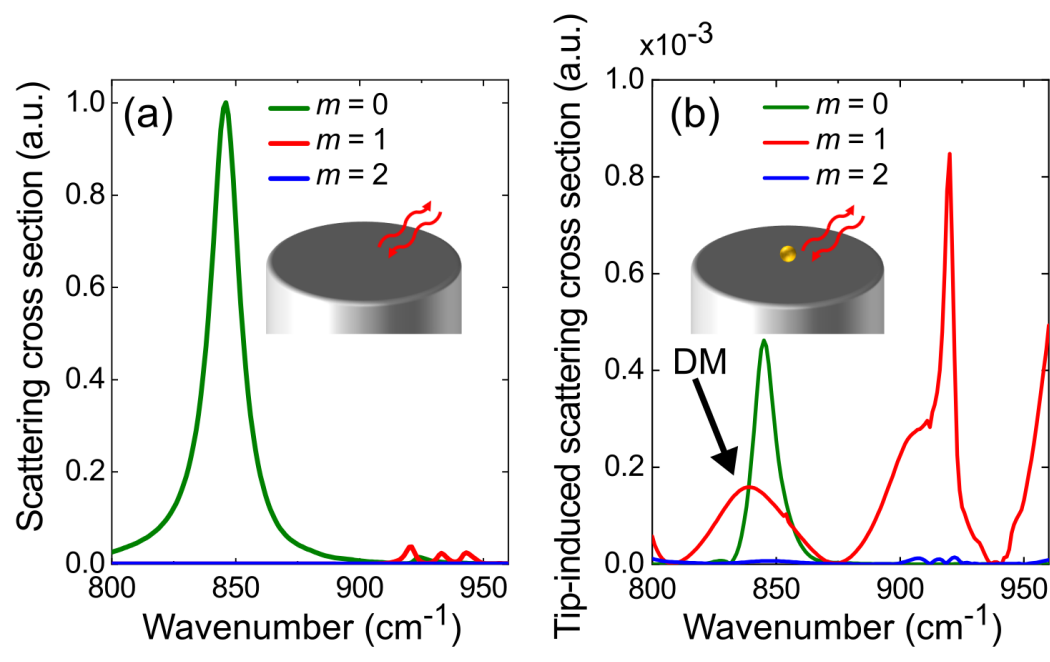

Monopole (M)

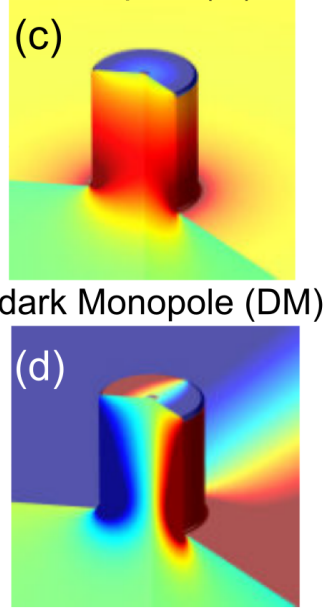

Figure 3: (a) Simulated scattering cross section of a single isolated pillar on a SiC substrate. The different colors separate the scattering contribution from modes with different azimuthal symmetry $m$. (b) Simulated tip-induced scattering cross section obtained by subtracting the far-field background. This procedure highlights the excitation of the dark monopole (DM, indicated with yellow dots in Figure 2) activated by the introduction of the tip (modeled by a small metallic sphere). Map of the surface normal field at the frequency of the monopole (c) and of the dark monopole (d).

Accurately simulating the experimental system is a complex task. Simulating the local illumination from the tip and the periodic nanopillar array would come at high computational expense. The exact shape of the AFM tip is a relative unknown, affecting both excitation of nanopillar modes and how they radiate to the far-field detector. In order to qualitatively 
investigate the effect of the tip in inducing the dark monopole mode observed in Figure 2 (b) we model the tip as a gold sphere with radius $r=30 \mathrm{~nm}$, placed $5 \mathrm{~nm}$ above a single isolated pillar. To simulate the demodulation procedure at higher harmonics of the tapping frequency $n \Omega$ (which ensures that only light which has interacted at least once with the tip is recorded in the far-field), we first solve for the pillar in the absence of the sphere with plane wave excitation. We then we use this field as a background for the full simulation including the sphere. Doing this we isolate the tip-induced scattering from the far-field. In Figure 3 (a) the far-field scattering cross section of a single pillar is shown, while in Figure 3 (b) the tip-induced scattering cross section resulting from the background subtraction procedure is reported. Spectra in Figures 3 (a), (b) are normalized to the maximum of the isolated pillar scattering cross section. The scattering contributions are divided by their azimuthal symmetry order, so that it is easy to distinguish the monopolar mode with $m=0$ symmetry from the transverse dipolar modes with $m=1$ symmetry. The spectra in Figure 3 (b) show that on the red side of the monopolar mode, another peak with $m=1$ symmetry appears, which is absent in far-field measurements. We associate this peak with the dark monopolar mode indicated by yellow dots in the experimental data of Figure 2 (b). In Figure 3 (c), (d) the normal component of the field in the presence of the metallic sphere is shown for the monopolar and dark monopolar modes, respectively. The dark monopole is polarized out of plane like the monopolar mode, but switches polarization along the pillar axis, meaning it has zero net dipole moment and does not couple to the far-field. From Figure 3 (b) it can be seen that another peak, slightly redshifted from the first transverse dipole, is also induced by the presence of the tip. This additional resonance arises from the coupling of the first transverse dipolar mode with the dipole induced by the tip, but it seems to have a lesser impact on the measured spectra, as no clear additional resonance close to the first transverse dipolar mode is seen experimentally in Figure 2 (a) or 2 (b).

Finally, to obtain the spatially resolved near-field distribution we perform a point by point measurement by continuously scanning the tip, either on top of a pillar or off pillar. 


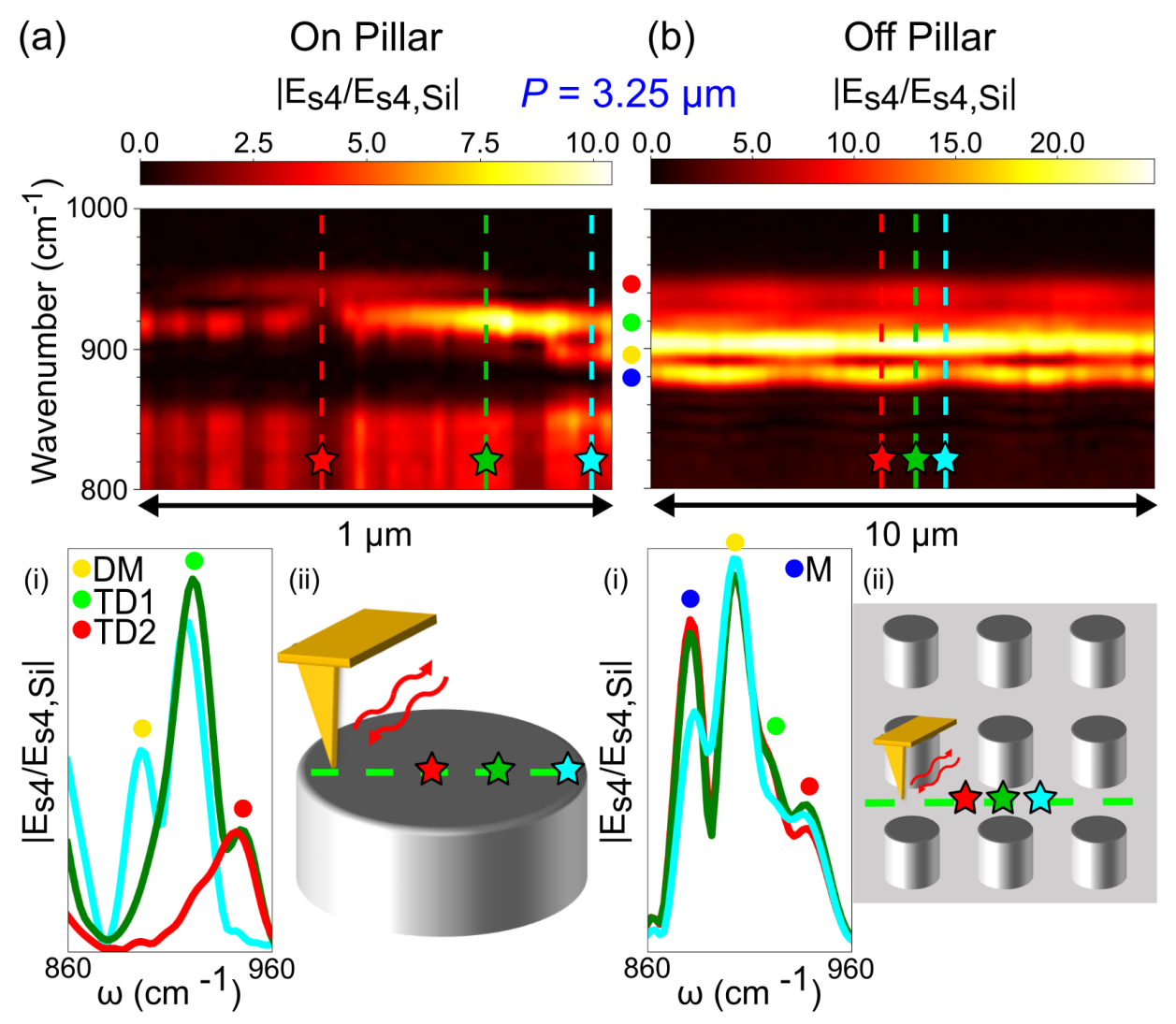

Figure 4: Colorplots of the near-field spectra collected as a function of tip position when scanning on top of a pillar (a), or on the substrate between pillars (b). In (i) three selected spectra showing variations of spectral response in different positions are shown. In (ii) sketch of the line along wich the data where collected. The stars represent the positions at which the spectra in (i) are taken.

We report in Figure 4 measurements obtained in this way for the array with $P=3.25 \mu \mathrm{m}$. In the insets (ii), the scan direction, highlighted with a green dashed line, is plotted on top of a sketch of the corresponding portion of the sample. In (i) we plot three spectra obtained at different positions, marked with three stars of different colors in both AFM images and colorplots. From Figure 4 (a), we see that the TD2 mode (red dot) is efficiently excited only when the tip is in the middle of the pillar, while as we expect, the TD1 mode (green dot) shows higher intensity when measuring on the edges of the pillar and completely disappears close to the center, in the point marked by the red star. Referring to the simulated field enhancements of Figures 1 (d-f), the monopole is expected to have high intensity only on the very edge of the pillar, which is hard to experimentally probe due to the high geometrical 
curvature of the region, leading to difficult sample-tip mechanical interaction. The TD2 resonance is not expected to have a maximum at the center of the structure (see Figure $1 \mathrm{f}$ ) as experimentally reported, showing that the tip interaction heavily modifies the field distribution of this mode. The asymmetry of Figure 4 (a) with respect to the pillar center can be attributed to the tilted illumination, which breaks the symmetry of the structure. Interestingly, we detect the presence of an additional mode only when measuring close to the side of the pillar. By comparing its frequency with the off pillar measurements of Figure 2 (b), we can identify this peak as the dark monopolar mode. This resonance is detected only on the side facing towards the illumination direction (from the right top in the colorplot and in (ii)). This asymmetry is consistent with previous studies of the perturbation introduced by the presence of an AFM tip on top of resonating antennas. ${ }^{62}$ To further confirm the spatial pattern of the modes measured in Figure 4 (a), we also perform hyperspectral imaging on top of one of the pillars (see Supporting Information). We find that integrating the signal over two different frequency ranges confirms that the TD1 mode shows higher response on the edges, while TD2 is stronger in the center.

The colorplot in Figure 4 (b) shows that the response of the substrate in between pillars varies weakly with small intensity modulations. The response of the TD1 and TD2 modes is found to be stronger when scanning close to the base of a pillar, on the side facing towards the illumination source. The intensity of the monopolar mode follows instead an opposite trend, peaking for points equidistant from adjacent pillars. As expected from the simulated field distributions of 1 (d-f), the response of the monopolar mode is stronger than that of the TD1 and TD2 modes when measuring off pillar. The weak frequency shifts seen at different positions in both Figures 4 (a), (b) can be at least partially attributed to coupling with the tip, as the use of conventional nano-FTIR tips with larger curvature radius increases these shifts (see Supporting Information). 


\section{Conclusions}

In conclusion, we report on the near-field study of the SPhPs spectral response of SiC pillar resonators in the mid-IR by means of sSNOM. These results are of critical importance for enhancing the efficiency of mid-IR emitters or for manipulation of thermal emission at the nanoscale with phonon-polariton antennas. As the near-field spectral response of a system supporting surface wave excitation can be in general different from the response measured in the far-field, this study will help in the understanding of further experiments where $\mathrm{SiC}$ resonators are coupled to other systems through near-field interaction. We further employ 3D electromagnetic simulations to understand the effect of the sSNOM tip in modifying the

response of the SPhPs antennas. Even though such effects are not related purely to the near-field response of the antennas, similar perturbations can be expected to occur when coupling the SPhPs resonators with other strong IR emitters or resonators.

\section{Acknowledgements}

The authors acknowledge the Deutsche Forschungsgemeinschaft (Clusters of Excellence: NanoInitiative Munich and e-Conversion, EXC 2089/1 - 390776260), the Bavarian Solar Energies Go Hybrid (SolTech) program and the Center for NanoScience (CeNS). E.C. acknowledges the European Commission for the ERC starting Grant 802989 CATALIGHT. S.D.L. is a Royal Society Research Fellow. S.D.L. and C.G. acknowledge support from the Royal Society grant RGFÉA181001. Y.L. acknowledges the funding received from the European Unions Framework Programme for Research and Innovation Horizon 2020 (20142020) under the Marie Skodowska-Curie Grant Agreement No. 754388 (LMU Research Fellows) and from LMUexcellent as part of LMU Munichs funding as University of Excellence within the frame-

work of the German Excellence Strategy. We acknowledge support from the Southampton Nanofabrication Centre. 


\section{References}

(1) Maier, S. A. Plasmonics: fundamentals and applications; Springer Science \& Business Media, 2007.

(2) Schuller, J. A.; Barnard, E. S.; Cai, W.; Jun, Y. C.; White, J. S.; Brongersma, M. L. Plasmonics for extreme light concentration and manipulation. Nature materials 2010, 9, 193.

(3) Atwater, H. A.; Polman, A. Materials For Sustainable Energy: A Collection of PeerReviewed Research and Review Articles from Nature Publishing Group; World Scientific, 2011; pp 1-11.

(4) Prikulis, J.; Svedberg, F.; Käll, M.; Enger, J.; Ramser, K.; Goksör, M.; Hanstorp, D. Optical spectroscopy of single trapped metal nanoparticles in solution. Nano Letters 2004, 4, 115-118.

(5) Njoki, P. N.; Lim, I.-I. S.; Mott, D.; Park, H.-Y.; Khan, B.; Mishra, S.; Sujakumar, R.; Luo, J.; Zhong, C.-J. Size correlation of optical and spectroscopic properties for gold nanoparticles. The Journal of Physical Chemistry C 2007, 111, 14664-14669.

(6) Campillo, I.; Pitarke, J.; Rubio, A.; Zarate, E.; Echenique, P. Inelastic lifetimes of hot electrons in real metals. Physical review letters 1999, 83, 2230.

(7) Brongersma, M. L.; Halas, N. J.; Nordlander, P. Plasmon-induced hot carrier science and technology. Nature nanotechnology 2015, 10, 25.

(8) Brown, A. M.; Sundararaman, R.; Narang, P.; Goddard III, W. A.; Atwater, H. A. Nonradiative plasmon decay and hot carrier dynamics: effects of phonons, surfaces, and geometry. Acs Nano 2015, 10, 957-966.

(9) Besteiro, L. V.; Kong, X.-T.; Wang, Z.; Hartland, G.; Govorov, A. O. Understanding 
hot-electron generation and plasmon relaxation in metal nanocrystals: Quantum and classical mechanisms. Acs Photonics 2017, 4, 2759-2781.

(10) Maier, S. A.; Kik, P. G.; Atwater, H. A.; Meltzer, S.; Harel, E.; Koel, B. E.; Requicha, A. A. Local detection of electromagnetic energy transport below the diffraction limit in metal nanoparticle plasmon waveguides. Nature materials 2003, 2, 229.

(11) Lal, S.; Hafner, J. H.; Halas, N. J.; Link, S.; Nordlander, P. Noble metal nanowires: from plasmon waveguides to passive and active devices. Accounts of Chemical Research 2012, 45, 1887-1895.

(12) Krauss, E.; Razinskas, G.; Köck, D.; Grossmann, S.; Hecht, B. Reversible Mapping and Sorting the Spin of Photons on the Nanoscale: A Spin-Optical Nano Device. Nano letters 2019,

(13) Prämassing, M.; Liebtrau, M.; Schill, H.; Irsen, S.; Linden, S. Interferometric near-field characterization of plasmonic slot waveguides in single-and poly-crystalline gold films. arXiv preprint arXiv:1907.06695 2019,

(14) Zhao, Y.; Belkin, M. A.; Alù, A. Twisted optical metamaterials for planarized ultrathin broadband circular polarizers. Nature communications 2012, 3, 870.

(15) Yu, N.; Capasso, F. Flat optics with designer metasurfaces. Nature materials 2014, 13, $139-150$.

(16) Ren, H.; Li, X.; Zhang, Q.; Gu, M. On-chip noninterference angular momentum multiplexing of broadband light. Science 2016, 352, 805-809.

(17) Sorger, V. J.; Oulton, R. F.; Ma, R.-M.; Zhang, X. Toward integrated plasmonic circuits. MRS bulletin 2012, 37, 728-738.

(18) Davis, T. J.; Gómez, D. E.; Roberts, A. Plasmonic circuits for manipulating optical information. 2017, 
(19) De Leon, N. P.; Lukin, M. D.; Park, H. Quantum plasmonic circuits. IEEE Journal of Selected Topics in Quantum Electronics 2012, 18, 1781-1791.

(20) Heeres, R. W.; Kouwenhoven, L. P.; Zwiller, V. Quantum interference in plasmonic circuits. Nature nanotechnology 2013, 8, 719.

(21) Khurgin, J. B. How to deal with the loss in plasmonics and metamaterials. Nature nanotechnology 2015, 10, 2.

(22) Baffou, G.; Girard, C.; Quidant, R. Mapping heat origin in plasmonic structures. Physical review letters 2010, 104, 136805.

(23) Baffou, G.; Quidant, R. Thermo-plasmonics: using metallic nanostructures as nanosources of heat. Laser \& Photonics Reviews 2013, \%, 171-187.

(24) Gobin, A. M.; Lee, M. H.; Halas, N. J.; James, W. D.; Drezek, R. A.; West, J. L. Nearinfrared resonant nanoshells for combined optical imaging and photothermal cancer therapy. Nano letters 2007, \%, 1929-1934.

(25) Zhou, L.; Zhang, C.; McClain, M. J.; Manjavacas, A.; Krauter, C. M.; Tian, S.; Berg, F.; Everitt, H. O.; Carter, E. A.; Nordlander, P., et al. Aluminum nanocrystals as a plasmonic photocatalyst for hydrogen dissociation. Nano letters 2016, 16, 1478-1484.

(26) Cortés, E. Activating plasmonic chemistry. Science 2018, 362, 28-29.

(27) Gargiulo, J.; Berte, R.; Li, Y.; Maier, S. A.; Cortes, E. From Optical to Chemical Hot Spots in Plasmonics. Accounts of chemical research 2019, 52, 2525-2535.

(28) Law, S.; Yu, L.; Rosenberg, A.; Wasserman, D. All-semiconductor plasmonic nanoantennas for infrared sensing. Nano letters 2013, 13, 4569-4574.

(29) Baldassarre, L.; Sakat, E.; Frigerio, J.; Samarelli, A.; Gallacher, K.; Calandrini, E.; Isella, G.; Paul, D. J.; Ortolani, M.; Biagioni, P. Midinfrared plasmon-enhanced spec- 
troscopy with germanium antennas on silicon substrates. Nano letters 2015, 15, 72257231.

(30) Biagioni, P.; Frigerio, J.; Samarelli, A.; Gallacher, K.; Baldassarre, L.; Sakat, E.; Calandrini, E.; Millar, R. W.; Giliberti, V.; Isella, G., et al. Group-IV midinfrared plasmonics. Journal of Nanophotonics 2015, 9, 093789.

(31) Koppens, F. H.; Chang, D. E.; Garcia de Abajo, F. J. Graphene plasmonics: a platform for strong light--matter interactions. Nano letters 2011, 11, 3370-3377.

(32) Grigorenko, A.; Polini, M.; Novoselov, K. Graphene plasmonics. Nature photonics 2012, 6,749 .

(33) Naik, G. V.; Kim, J.; Boltasseva, A. Oxides and nitrides as alternative plasmonic materials in the optical range. Optical Materials Express 2011, 1, 1090-1099.

(34) Babicheva, V. E.; Boltasseva, A.; Lavrinenko, A. V. Transparent conducting oxides for electro-optical plasmonic modulators. Nanophotonics 2015, 4, 165-185.

(35) Boltasseva, A.; Atwater, H. A. Low-loss plasmonic metamaterials. Science 2011, 331, $290-291$.

(36) Caldwell, J. D.; Lindsay, L.; Giannini, V.; Vurgaftman, I.; Reinecke, T. L.; Maier, S. A.; Glembocki, O. J. Low-loss, infrared and terahertz nanophotonics using surface phonon polaritons. Nanophotonics 2015, 4, 44-68.

(37) Neubrech, F.; Pucci, A.; Cornelius, T. W.; Karim, S.; García-Etxarri, A.; Aizpurua, J. Resonant plasmonic and vibrational coupling in a tailored nanoantenna for infrared detection. Physical review letters 2008, 101, 157403.

(38) Tittl, A.; Leitis, A.; Liu, M.; Yesilkoy, F.; Choi, D.-Y.; Neshev, D. N.; Kivshar, Y. S.; Altug, H. Imaging-based molecular barcoding with pixelated dielectric metasurfaces. Science 2018, 360, 1105-1109. 
(39) Neubrech, F.; Weber, D.; Enders, D.; Nagao, T.; Pucci, A. Antenna sensing of surface phonon polaritons. The Journal of Physical Chemistry C 2010, 114, 7299-7301.

(40) Mutschke, H.; Andersen, A.; Clément, D.; Henning, T.; Peiter, G. Infrared properties of $\mathrm{SiC}$ particles. arXiv preprint astro-ph/9903031 1999,

(41) Anderson, M. S. Enhanced infrared absorption with dielectric nanoparticles. Applied physics letters 2003, 83, 2964-2966.

(42) Berte, R.; Gubbin, C. R.; Wheeler, V. D.; Giles, A. J.; Giannini, V.; Maier, S. A.; De Liberato, S.; Caldwell, J. D. Sub-nanometer thin oxide film sensing with localized surface phonon polaritons. ACS Photonics 2018, 5, 2807-2815.

(43) Autore, M.; Li, P.; Dolado, I.; Alfaro-Mozaz, F. J.; Esteban, R.; Atxabal, A.; Casanova, F.; Hueso, L. E.; Alonso-González, P.; Aizpurua, J., et al. Boron nitride nanoresonators for phonon-enhanced molecular vibrational spectroscopy at the strong coupling limit. Light: Science \& Applications 2018, \%, 17172.

(44) Shchegrov, A. V.; Joulain, K.; Carminati, R.; Greffet, J.-J. Near-field spectral effects due to electromagnetic surface excitations. Physical Review Letters 2000, 85, 1548.

(45) Greffet, J.-J.; Carminati, R.; Joulain, K.; Mulet, J.-P.; Mainguy, S.; Chen, Y. Coherent emission of light by thermal sources. Nature 2002, 416, 61.

(46) Schuller, J. A.; Taubner, T.; Brongersma, M. L. Optical antenna thermal emitters. Nature Photonics 2009, 3, 658.

(47) Shen, S.; Narayanaswamy, A.; Chen, G. Surface phonon polaritons mediated energy transfer between nanoscale gaps. Nano letters 2009, 9, 2909-2913.

(48) Wang, T.; Li, P.; Chigrin, D. N.; Giles, A. J.; Bezares, F. J.; Glembocki, O. J.; Caldwell, J. D.; Taubner, T. Phonon-polaritonic bowtie nanoantennas: controlling infrared thermal radiation at the nanoscale. ACS Photonics 2017, 4, 1753-1760. 
(49) Hillenbrand, R.; Taubner, T.; Keilmann, F. Phonon-enhanced light-matter interaction at the nanometre scale. Nature 2002, 418, 159.

(50) Alfaro-Mozaz, F.; Alonso-González, P.; Vélez, S.; Dolado, I.; Autore, M.; Mastel, S.; Casanova, F.; Hueso, L.; Li, P.; Nikitin, A. Y., et al. Nanoimaging of resonating hyperbolic polaritons in linear boron nitride antennas. Nature communications 2017, 8, 15624 .

(51) Phillips, C.; Gilburd, L.; Xu, X. G.; Walker, G. C. Surface and Volume Phonon Polaritons in Boron Nitride Nanotubes. The Journal of Physical Chemistry Letters 2019, $10,4851-4856$.

(52) Caldwell, J. D.; Glembocki, O. J.; Francescato, Y.; Sharac, N.; Giannini, V.; Bezares, F. J.; Long, J. P.; Owrutsky, J. C.; Vurgaftman, I.; Tischler, J. G., et al. Low-loss, extreme subdiffraction photon confinement via silicon carbide localized surface phonon polariton resonators. Nano letters 2013, 13, 3690-3697.

(53) Gubbin, C. R.; Maier, S. A.; De Liberato, S. Theoretical investigation of phonon polaritons in SiC micropillar resonators. Physical Review B 2017, 95, 035313.

(54) Chen, Y.; Francescato, Y.; Caldwell, J. D.; Giannini, V.; Maß, T. W.; Glembocki, O. J.; Bezares, F. J.; Taubner, T.; Kasica, R.; Hong, M., et al. Spectral tuning of localized surface phonon polariton resonators for low-loss mid-IR applications. Acs Photonics 2014, 1, 718-724.

(55) Gubbin, C. R.; Martini, F.; Politi, A.; Maier, S. A.; De Liberato, S. Strong and coherent coupling between localized and propagating phonon polaritons. Physical Review Letters 2016, 116, 246402.

(56) Gubbin, C. R.; De Liberato, S. Theory of nonlinear polaritonics: $\chi(2)$ scattering on a $\beta$-SiC surface. ACS Photonics 2017, 4, 1381-1388. 
(57) Razdolski, I.; Passler, N. C.; Gubbin, C. R.; Winta, C. J.; Cernansky, R.; Martini, F.; Politi, A.; Maier, S. A.; Wolf, M.; Paarmann, A., et al. Second harmonic generation from strongly coupled localized and propagating phonon-polariton modes. Physical Review B 2018, 98, 125425 .

(58) Gubbin, C. R.; De Liberato, S. Theory of four-wave-mixing in phonon polaritons. ACS Photonics 2018, 5, 284-288.

(59) Dunkelberger, A. D.; Ellis, C. T.; Ratchford, D. C.; Giles, A. J.; Kim, M.; Kim, C. S.; Spann, B. T.; Vurgaftman, I.; Tischler, J. G.; Long, J. P., et al. Active tuning of surface phonon polariton resonances via carrier photoinjection. Nature Photonics 2018, 12, 5056.

(60) Nordlander, P.; Prodan, E. Plasmon hybridization in nanoparticles near metallic surfaces. Nano Letters 2004, 4, 2209-2213.

(61) Knoll, B.; Keilmann, F. Enhanced dielectric contrast in scattering-type scanning nearfield optical microscopy. Optics communications 2000, 182, 321-328.

(62) García-Etxarri, A.; Romero, I.; de Abajo, F. J. G.; Hillenbrand, R.; Aizpurua, J. Influence of the tip in near-field imaging of nanoparticle plasmonic modes: Weak and strong coupling regimes. Physical Review B 2009, 79, 125439. 